\title{
Patients with bipolar disorders share similar but attenuated prospective memory impairments with patients with schizophrenia
}

\author{
R. C. K. Chan ${ }^{1 *}$, S. S. Y. Lui ${ }^{1,2,3}$, Y. Wang ${ }^{1}$, A. C. Y. Liu ${ }^{3}$, W. W. H. Chui ${ }^{3}$, D. H. K. Shum ${ }^{4}$ \\ and E. F. C. Cheung ${ }^{3}$ \\ ${ }^{1}$ Neuropsychology and Applied Cognitive Neuroscience Laboratory, Key Laboratory of Mental Health, Institute of Psychology, Chinese Academy of \\ Sciences, Beijing, People's Republic of China \\ ${ }^{2}$ University of Chinese Academy of Sciences, Beijing, People's Republic of China \\ ${ }^{3}$ Castle Peak Hospital, Hong Kong Special Administrative Region, People's Republic of China \\ ${ }^{4}$ Behavioural Basis of Health Research Program, Griffith Health Institute, Griffith University, Gold Coast, Australia
}

Background. Prospective memory (PM) refers to the ability to remember to carry out an intended action in the future. PM is consistently found to be impaired in individuals with schizophrenia. Bipolar disorder and schizophrenia may represent conditions along a continuum, and share similar neurocognitive and genetic architecture. This study aimed to compare the nature and extent of PM impairment in individuals with schizophrenia and bipolar disorder.

Method. Participants were 38 out-patients with schizophrenia and 40 out-patients with bipolar disorder in an early psychosis intervention programme, and 37 healthy controls. Time-, event- and activity-based PMs were assessed using a dual-task laboratory paradigm. Self-reported PM performance was gauged using the Prospective and Retrospective Memory Questionnaire. Analysis of covariance (ANCOVA), with intelligence quotient (IQ) and education included as covariates, was used to examine group difference on various types of PM. Repeated measures of ANCOVA were used to examine the group $\times$ PM type interaction effect. Correspondence between laboratory and self-reported PM measures was examined using correlational analysis.

Results. The group $\times$ PM type interaction effect was not significant, but the main effect of group was significant. Patients with schizophrenia and patients with bipolar disorder both performed more poorly than healthy participants in PM. The two clinical groups did not significantly differ in PM. Laboratory and self-reported PM measures did not correlate significantly with each other.

Conclusions. Patients with bipolar disorder shared a similar PM impairment with those with schizophrenia. Findings of this study extended the similarity in neurocognitive impairments between the two psychiatric disorders to PM.

Received 10 May 2012; Revised 27 August 2012; Accepted 30 August 2012; First published online 26 October 2012

Key words: Bipolar disorder, prospective memory, schizophrenia.

\section{Introduction}

Memory is an essential and unique cognitive function in humans. It is usually classified into subtypes such as working memory, short-term memory and longterm memory. However, prior classification models of memory are concerned only with the ability to remember things that happened in the past. Prospective memory (PM), the ability to remember to carry out intentions in the future, has attracted considerable

* Address for correspondence: Professor R. C. K. Chan, Institute of Psychology, Chinese Academy of Sciences, 4A Datun Road, Beijing 100101, People's Republic of China.

(Email : rckchan@psych.ac.cn) interest in the past three decades (Kvavilashvili, 1992). The growth of interest could be understood in the light of the close relationship between PM and everyday functioning. PM is usually classified into time-based PM (e.g. to remember to attend a meeting at 15:00 hours), event-based PM (e.g. to remember to pass on a message when a classmate shows up) and activity-based PM (e.g. to remember to switch off the light before leaving the office). These classifications are made based on the nature of the cues associated with the intended action (Einstein \& McDaniel, 1990; Kvavilashvili \& Ellis, 1996). Ellis (1996) proposed that PM involves five stages: (1) encoding the intention; (2) maintenance of the intention; (3) detection of cues and retrieval of the intention; (4) execution 
of action; and (5) evaluation of the outcome. PM is usually studied using a dual-task paradigm (Einstein \& McDaniel, 1996), during which a participant is engaged in an ongoing task during the time lapse between the encoding stage and execution stage of a PM task, and he has to retrieve and execute a delayed intention at a specific time or at the appearance of a specific cue. Apart from laboratory assessments, PM functions could also be evaluated by self-reported questionnaires, which commonly ask a respondent to report the frequency of forgetting on a Likert scale. This method of evaluation has the benefit of estimating one's everyday PM performance, but such selfestimation might not necessarily be accurate.

Early PM studies usually involve comparing the performance of PM in older and younger individuals and manipulating task-related variables such as cue saliency and number of cues (Einstein \& McDaniel, 1990; Zeintl et al. 2007; Kliegel et al. 2008b). More recently, Kliegel et al. $(2008 a, 2011)$ noted a shift of research focus to include the study of PM in various clinical populations such as patients with schizophrenia. This is mainly because clinical patients are commonly found by their significant others or case managers to have prospective forgetting and that these problems are affecting their everyday independent functioning. In a recent meta-analysis, Wang et al. (2009) identified 11 PM studies in schizophrenia and found that they all recruited patients with long duration of illness (DOI), with a range from 4.7 to 26.1 years. More recently, two studies have recruited patients with shorter DOI (Lui et al. 2011; Zhou et al. 2012) and suggested that PM is impaired in patients with schizophrenia regardless of disease chronicity. Compared with PM research in schizophrenia, the study of PM in another major mental health disorder, bipolar disorder, is still in its infancy. To date there has been only one study (Lee et al. 2010) that showed that individuals with bipolar disorder are impaired in PM.

To examine whether PM is a neuropsychological marker for psychosis, a few studies have recruited non-clinical samples that are at risk of developing psychosis (Wang et al. 2008b, 2010; Lui et al. 2011). Wang et al. (2008b) found that individuals with schizotypal personality features are impaired in PM functions. The findings, however, were inconsistent for those who are genetically at risk of developing psychosis. While Wang et al. (2010) found significant PM impairments in non-psychotic first-degree relatives, Lui et al. (2011) did not find PM impairments in nonpsychotic siblings of patients with schizophrenia.

While it is acknowledged that the empirical evidence for PM as a neuropsychological marker is far from conclusive, there is another line of promising research in the PM and mental health area. Contrary to the traditional Kraepelinian view of dichotomy, there is a substantial body of literature that shows that schizophrenia and bipolar disorder share remarkable commonalities. A variety of neurocognitive dysfunctions, in particular sustained attention (Braff, 1993; Chan et al. 2006a, b; Arts et al. 2008), verbal and visual memory (Heaton et al. 2001; Chan et al. 2006a; Hill et al. 2008), working memory (Gold et al. 1997; Chan et al. 2006b; Glahn et al. 2007; Arts et al. 2008) and executive functions (Chan et al. 2004; Glahn et al. 2007), has been demonstrated in both disorders. Moreover, empirical findings from genetics, electrophysiology and neuroimaging (Shifman et al. 2004; Potash \& Bienvenu, 2009; Ellison-Wright \& Bullmore, 2010; Ivleva et al. 2010; Yu et al. 2010) have also converged to support the concept of unitary psychosis or continuum of psychosis (Crow, 1986; Linscott \& van Os, 2010).

We based this study on the concept of continuum of psychosis and hypothesized that patients with schizophrenia and bipolar disorder would share similar PM impairments. In addition, we hypothesized that patients with bipolar disorder would have a milder PM impairment than patients with schizophrenia. To test our hypotheses, we conducted a comparative study by recruiting both groups of patients and examining their performance on an identical dual-task laboratory PM paradigm. To ensure that the groups were more directly comparable, we recruited the two groups of patients from an early psychosis intervention programme where they had similar DOI and shorter disease chronicity, and received similar medication prescription regimens.

\section{Method \\ Participants}

A total of 38 stable out-patients with an International Classification of Diseases, Tenth Revision (ICD-10; WHO, 1992) diagnosis of schizophrenia and 40 outpatients with an ICD-10 diagnosis of bipolar disorder (22 with unipolar mania) were recruited from an early psychosis intervention programme in Hong Kong. The diagnoses were ascertained in clinical interviews by two qualified psychiatrists supplemented by information from medical records. Exclusion criteria were: (1) lifetime history of substance abuse (ICD-10); (2) history of electroconvulsive therapy in the past 6 months; (3) history of neurological disorder; (4) history of head injury with loss of consciousness for more than $30 \mathrm{~min}$; and (5) mental retardation. As controls, 37 healthy participants were recruited from nursing schools and youth centres in the same local area as the intervention programme. All the healthy participants 
were interviewed by a qualified psychiatrist to ensure the absence of lifetime and family history of psychosis. The sample with schizophrenia $(n=38)$ had previously participated in another PM study and the findings have been reported elsewhere (Lui et al. 2011). The sample with bipolar disorder $(n=40)$ had never participated in another PM study. All participants were introduced to the study and invited to ask questions before signing a consent document. The study was approved by the Ethics Committee of the New Territories West Cluster of Hospital Authority in Hong Kong.

The demographics and psychiatric history of the two clinical samples were retrieved from medical records. The current dosage of antipsychotic medication was expressed in terms of the percentage of maximum British National Formulary (BNF)recommended dose (British Medical Association and Royal Pharmaceutical Society of Great Britain, 2009) rather than chlorpromazine-equivalent dosage. This is because the majority of participants received second-generation antipsychotic (SGA) rather than first-generation antipsychotic (FGA) medications (Hung, 2007). Patients with schizophrenia and bipolar disorder were assessed using the Positive and Negative Symptom Scale (PANSS; Kay et al. 1987). The Young Mania Rating Scale (Young et al. 1978) and the Hamilton Depression Rating Scales (HAMD; Hamilton, 1960) were additionally used in the sample with bipolar disorder. Intellectual functioning was estimated by using a pro-rating method based on the arithmetic, similarity and digit span subscales of the Chinese version of the Wechsler Adult Intelligence Scale-Revised (Gong et al. 1992). All participants were assessed with the Annett Handedness Scale (Spreen \& Strauss, 1991).

\section{Characteristics of participants}

The three groups of participants did not differ in age, gender and handedness. However, group differences in education and intelligence quotient (IQ) were significant $\left(F_{2,112}=7.742, p=0.001 ; F_{2,112}=6.263, p=0.003\right)$. The average DOIs for patients with schizophrenia and bipolar disorder were 19.8 (s.D. =11.5) months and 18.4 (S.D. $=19.8)$ months, respectively, and they were both at the early course of their illness and not significantly different $\left(F_{1,72}=0.157, p=0.693\right)$. The participants in the two clinical groups had low prevalence of positive and negative symptoms, as measured by the PANSS. The patients with bipolar disorder were clinically euthymic, as measured by the YMS and HAMD (Table 1).

Regarding current antipsychotic medications, three patients (not medication-naive) were not receiving any psychiatric medications at the time of assessments, nine were receiving FGAs, and 53 were receiving SGAs. A total of 26 patients with bipolar disorder and four patients with schizophrenia were receiving mood stabilizers (lithium, valproate or lamotrigine); nine patients with schizophrenia and four patients with bipolar disorder were receiving antidepressants. In all, 17 patients with schizophrenia and 12 patients with bipolar disorder were receiving anti-cholinergic medications at doses ranging from $2 \mathrm{mg}$ to $8 \mathrm{mg}$ daily; four patients with schizophrenia and three patients with bipolar disorder were receiving benzodiazepines at doses of lorazepam equivalents ranging from $0.5 \mathrm{mg}$ to $2 \mathrm{mg}$ daily.

\section{Laboratory PM assessment}

A dual-task (Einstein \& McDaniel, 1996) computerized paradigm was developed by the authors (Wang et al. $2008 b$ ) to measure time-, event- and activity-based PM. It comprised four sessions; each required participants to perform a delayed intention at a specific time or at the appearance of a specific cue, while continuously engaged in an ongoing task. Details of our paradigm have been described elsewhere (Lui et al. 2011).

There were two sessions to measure event-based PM. In the semantic event-based PM session, a series of four-character phrases in Chinese was sequentially presented on the computer screen at a rate of $4 \mathrm{~s}$, and the participants were asked to judge whether the phrases were idioms or not (i.e. the ongoing task) by pressing pre-specified response buttons. There were 88 trials in the ongoing task for this session. At the same time, participants were asked before the start of the ongoing task to press another pre-specified button when they saw the appearance of an animal character (e.g. monkey) in the phrases (i.e. the PM task). There were a total of five PM targets irregularly presented throughout the approximately 6-min semantic eventbased PM session. In the perceptual event-based PM session, a similar dual-task paradigm was used. However, the 122 trials in the ongoing task involved asking participants to judge whether a perceptually degraded digit that appeared on the screen was the digit 0 , and the PM task involved asking them to press another specified button when they saw an arrow underneath the degraded digits. There were a total of five PM targets irregularly presented to the participants throughout this approximately 6-min session. The omission errors (i.e. failure to press the prespecified button when the PM cues appeared on the screen) for the PM task were recorded.

There were two sessions to measure time-based PM. The ongoing tasks for the semantic time-based 
Table 1. Descriptive summary of the sociodemographic and clinical variables of all participants

\begin{tabular}{|c|c|c|c|c|c|c|c|c|}
\hline \multirow[b]{2}{*}{ Mean age, years (S.D.) } & \multicolumn{2}{|c|}{ Healthy controls $(n=37)$} & \multicolumn{2}{|c|}{ Schizophrenia $(n=38)$} & \multicolumn{2}{|c|}{ Bipolar disorder $(n=40)$} & \multirow{3}{*}{$\begin{array}{l}F \text { or } \chi^{2} \text { statistic } \\
0.266 \\
2681^{a}\end{array}$} & \multirow{2}{*}{$\frac{p}{0.767}$} \\
\hline & 22.35 & (3.44) & 21.76 & $(4.35)$ & 22.38 & $(4.54)$ & & \\
\hline Gender, $n$ & & & & & & & & 0.262 \\
\hline Male & 10 & & 14 & & 18 & & & \\
\hline Female & 27 & & 24 & & 22 & & & \\
\hline Handedness, $n^{\mathrm{b}}$ & & & & & & & $0.248^{\mathrm{a}}$ & 0.883 \\
\hline Right & 35 & & 36 & & 36 & & & \\
\hline Left & 2 & & 2 & & 3 & & & \\
\hline Mean education, years (S.D.) & 14.59 & $(2.31)$ & 12.58 & $(2.60)$ & 12.78 & $(2.42)$ & 7.742 & 0.001 \\
\hline Mean IQ estimate (s.D.) & 110.19 & (6.99) & 101.82 & (13.53) & 109.80 & (13.26) & 6.263 & 0.003 \\
\hline Mean duration of illness, months (S.D.) & - & - & 19.84 & (11.45) & 18.36 & $(19.82)$ & 0.157 & 0.693 \\
\hline $\begin{array}{l}\text { Mean antipsychotic dose, \% of } \\
\text { maximum BNF dosage }{ }^{\text {c }} \text { (s.D.) }\end{array}$ & - & - & 49.61 & (31.52) & 17.12 & (23.63) & 26.283 & 0.0001 \\
\hline Mean PANSS positive (S.D.) & - & - & 10.74 & $(4.07)$ & 8.22 & (2.18) & 10.796 & 0.002 \\
\hline Mean PANSS negative (S.D.) & - & - & 15.53 & $(6.41)$ & 8.19 & (2.10) & 42.765 & 0.0001 \\
\hline Mean PANSS general (s.D.) & - & - & 23.63 & $(6.48)$ & 17.97 & (2.95) & 22.922 & 0.0001 \\
\hline Mean PANSS total (S.D.) & - & - & 49.89 & (15.76) & 34.39 & $(6.22)$ & 30.339 & 0.0001 \\
\hline Mean HAMD (s.D.) & - & - & - & - & 1.06 & (1.97) & - & - \\
\hline Mean YMRS (S.D.) & - & - & - & - & 2.36 & (2.58) & - & - \\
\hline
\end{tabular}

S.D., Standard deviation; IQ, intelligence quotient; BNF, British National Formulary ; PANSS, Positive and Negative Symptom Scale; HAMD, Hamilton Depression Rating Scale; YMRS, Young Mania Rating Scale.

${ }^{\mathrm{a}} \chi^{2}$ Test.

${ }^{\mathrm{b}}$ For one bipolar subject, information on handedness is missing.

${ }^{\mathrm{c}}$ Medications given in average percentage of maximum BNF-recommended dosage. 
PM session and the perceptual time-based PM session were identical to those of the tasks in the event-based PM sessions. The main difference is that the PM task in the time-based sessions required participants to monitor the time and to press a pre-specified button when the digital clock situated near the keyboard reached a full minute (e.g. 12:00:00). In each of the time-based sessions, there were five trials of PM task, each presented at a regular interval of $1 \mathrm{~min}$, and numerous (90 and 135) trials of the ongoing task. The omission errors (i.e. failure to press the pre-specified button when the clock reached a full minute) for the PM task were recorded.

In the event-based PM sessions, participants had shorter time limits $(1.8-2.8 \mathrm{~s}$ for the semantic event-based session and 3.0-4.0 s for the perceptual event-based session) to execute the PM task. In the time-based PM sessions, they had a longer time limit of $10 \mathrm{~s}$ to execute the PM task. Our paradigm did not specifically record how frequent participants monitored the elapsing time, or how many substitution errors (e.g. pressing a wrong button instead of the pre-specified button) they made. Notably, the clock was available in front of the participant all the time throughout the sessions.

There were four trials of activity-based PM. Participants were instructed in the beginning of the session that they should press a pre-specified button when each of the four PM sessions ended. Upon completion of each session, a phrase 'Thank you for your participation. Bye!' would appear on the screen. The measure of activity-based PM was the number of omission errors.

The PM raw scores for each of the four sessions were calculated by dividing the total number of correctly executed PM tasks by the total number of trials of the PM task. In other words, a PM raw score of 1 indicates that there is no omission error made whereas a PM raw score of 0.2 indicates that the participant correctly executed the PM task in one out of the five trials in a time-based or event-based PM session. Likewise, the raw score for activity-based PM was calculated by dividing the total number of correctly executed PM tasks (i.e. pressing a pre-specified button as the session ended) by the total number of trials (i.e. four trials).

\section{Self-reported PM performance}

The Prospective and Retrospective Memory Questionnaire (PRMQ; Smith et al. 2000), a 16-item selfreported questionnaire, was used. This questionnaire comprises eight items to estimate one's self-perception of time-based and event-based PM performance in everyday life, as well as eight items to estimate the retrospective memory performance.

\section{Data analysis}

Given that previous studies had consistently found significant correlations between education, IQ and PM performance (Wang et al. 2009), and the fact that there was a significant group difference in education and IQ in our study, we used a series of analyses of covariance (ANCOVAs) to examine group difference in time-, event- and activity-based PM raw scores, with education and IQ as covariates.

To estimate the effect size of the significant group difference, post-hoc pairwise comparison was used. To examine if the disease status of schizophrenia and bipolar disorder differentially affects various PM types, a repeated-measures 3 (group: schizophrenia, bipolar disorder, healthy controls) $\times 5$ (PM type: semantictime based PM, semantic event-based PM, perceptual event-based PM, perceptual time-based PM, activitybased PM) ANCOVA with IQ and education as covariates was conducted.

To examine the association between laboratory PM and self-reported PM performance, we standardized the raw scores of PM measures using $Z$ transformation. The composite score for the laboratory PM was the sum of semantic event-based, perceptual eventbased, semantic time-based, perceptual time-based and activity-based PM standardized scores. We then performed Spearman's (two-tailed) correlational analyses to examine the associations between the standardized composite laboratory PM score and the standardized PM subtotal score of the PRMQ, with education and IQ as covariates. The three groups of participants were analysed separately.

To examine the possible effect of medications on PM, we standardized the dosage of anti-cholinergic medication, benzodiazepines (in lorazepam equivalents) and antipsychotic medications (percentage of maximum BNF dose) using $Z$ transformations. Correlations between laboratory PM and dosage of different types of medications were examined using Spearman's (two-tailed) correlational analyses.

\section{Results}

Table 2 shows the PM performance for the three groups of participants. We found a significant group difference in semantic time-based PM $\left(F_{2,110}=3.583, p=0.031\right)$, after controlling for education and IQ. However, the group differences in semantic event-based $\operatorname{PM}\left(F_{2,110}=2.426, p=0.093\right)$, perceptual time-based PM $\left(F_{2,110}=0.995, \quad p=0.373\right)$, perceptual event-based PM $\left(F_{2,110}=2.450, p=0.091\right)$ and activity-based PM 
$\left(F_{2,110}=0.287, p=0.751\right)$ were not statistically significant.

Post-hoc pairwise comparison found that patients with schizophrenia and patients with bipolar disorder both performed more poorly than healthy participants in the semantic time-based PM $(p=0.011$ and $p=0.048$, respectively). However, the PM deficit in the schizophrenia group has a larger effect size (Cohen's $d=0.913$ ) than that of the bipolar disorder group (Cohen's $d=0.526)$. Post-hoc pairwise comparison found that patients with schizophrenia and patients with bipolar disorder had no statistically significant difference in semantic time-based PM $(p=0.497)$.

The $3 \times 5$ repeated-measures ANCOVA found that the group $\times \mathrm{PM}$ type interaction effect failed to reach statistical significance $\left(F_{8,440}=1.339, p=0.234\right.$, Greenhouse-Greisser). The main effect for group was statistically significant $\left(F_{2,110}=4.496, p=0.013\right.$, partial $\eta^{2}=0.076$ ).

Post-hoc pairwise comparison found that the group with schizophrenia, having a mean PM raw score (averaged across all PM types) of 0.821, performed more poorly in PM than the healthy group, which had a mean PM raw score of $0.918(p=0.004$, Cohen's $d=0.846$ ). Likewise, the group with bipolar disorder, having a mean PM raw score of 0.856 , also performed more poorly in PM than the healthy group ( $p=0.049$, Cohen's $d=0.502$ ). However, post-hoc pairwise comparison found that the two clinical groups did not differ in the mean PM raw score $(p=0.274)$.

The main effect of PM type was statistically significant $\left(F_{4,107}=24.356, p=<0.001\right.$, partial $\left.\eta^{2}=0.477\right)$. The mean PM raw scores (averaged across groups) in the semantic event-based PM session, semantic timebased PM session, perceptual event-based PM session, perceptual time-based PM session and activity-based PM session were $0.698,0.838,0.938,0.876$ and 0.976 , respectively. Post-hoc pairwise comparison found that the mean PM raw score of semantic event-based PM was significantly lower than that of the other four PM types $\left(p^{\prime} s<0.001\right)$. The mean score of semantic timebased PM was significantly lower than that of perceptual event-based and activity-based PM ( $p$ 's $<0.001)$. The mean score of perceptual time-based PM was significantly lower than that of perceptual event-based $(p=0.021)$ and activity-based PM $(p<0.001)$. The mean score of perceptual event-based PM was significantly lower than that of activity-based PM $(p=0.033)$. These findings suggested that the semantic event-based PM session was the most difficult among the five PM types.

We examined the correlation between laboratory PM, self-reported PM and medications in the three groups of participants separately. For patients with schizophrenia, patients with bipolar disorder and 
Table 3. Correlations between laboratory PM, self-reported PM and medications

\begin{tabular}{lll}
\hline & \multicolumn{2}{l}{ Laboratory PM } \\
\cline { 2 - 3 } & $\begin{array}{l}\text { Schizophrenia } \\
(n=38)\end{array}$ & $\begin{array}{l}\text { Bipolar disorder } \\
(n=40)\end{array}$ \\
& & \\
\hline Self-reported PM & 0.047 & -0.278 \\
$\quad$ Correlation coefficient: $r$ & 0.791 & 0.086 \\
$p$ & & \\
Antipsychotic medications & 0.001 & -0.187 \\
Correlation coefficient: $r$ & 0.997 & 0.253 \\
$p$ & & 0.023 \\
Anticholinergic medications & 0.059 & 0.890 \\
Correlation coefficient: $r$ & 0.723 & 0.272 \\
$p$ & & 0.094 \\
Benzodiazepine medications & -0.112 & \\
Correlation coefficient: $r$ & 0.502 & \\
$p$ & &
\end{tabular}

PM, Prospective memory.

healthy participants, none of the correlations was statistically significant, as shown in Table 3.

\section{Discussion}

This study is one the few that have directly compared PM performance in patients with schizophrenia with that in patients with bipolar disorder using an identical laboratory paradigm. Our findings suggest that there is an overall significant group difference in PM, and this was mainly contributed by the difference in semantic time-based PM. Using post-hoc analyses, we demonstrated that both patients with schizophrenia and patients with bipolar disorder performed more poorly in PM than healthy participants.

There is a growing body of empirical evidence to support the existence of PM impairment in schizophrenia (Shum et al. 2004; Altgassen et al. 2008; Wang et al. 2008a; for a review and meta-analysis, see Wang et al. 2009). The underlying cause of such a deficit is likely to be a reduction of cognitive resources available for monitoring task requirements in these individuals. The ability to monitor and maintain two intentions of action simultaneously, one for the ongoing task and the other for the PM task, is very essential in the dualtask PM paradigms. In such paradigms, participants have to continuously monitor two different intentions and actions, and allocate attention to varying demands of the tasks to successfully execute the PM task within a specified time limit. The 'supervisory attentional system' (SAS) model proposed by Norman \& Shallice (1986) defines an integrated set of cognitive processes that facilitates the timely allocation of attention to the demands of different, competing tasks in everyday life. Such cognitive processes have been consistently found to be impaired in individuals with schizophrenia (Burgess \& Shallice, 1996a, b; Heinrichs \& Zakzanis, 1998; Chan et al. 2004, 2006a, b).

Contrary to the growing PM research in schizophrenia, there have been few studies on PM in bipolar disorder and a shortage of empirical evidence in this area. Moreover, the previous findings (Lee et al. 2010) might also be confounded by disease chronicity $(\mathrm{DOI}=14.3$ years $)$ and long-term medication effect. Compared with the previous studies, our findings are likely to be more robust because our clinical groups had low prevalence of psychiatric symptoms, short DOI, and were less likely to be subject to medication effect. Though all our clinical participants were medicated, the negative results in correlations between PM and medications further reduced the concerns that our findings are confounded by the effects of medication. In fact, Tamlyn et al. (1992) reported that lorazepam at doses not higher than $2 \mathrm{mg}$ daily do not significantly affect memory or neurocognitive performance in patients with psychosis.

Though both clinical groups were impaired in PM, the magnitude of impairment as illustrated by the effect size found in schizophrenia was greater than that found in bipolar disorder. It is likely that no significant quantitative or qualitative difference in PM exists between the two clinical groups, as indicated by the fact that the group $\times \mathrm{PM}$ type interaction was not significant, and the post-hoc pairwise comparison of overall PM between the two clinical groups was also not significant. 
The current findings did not show significant correlation between laboratory PM assessment and selfreported PM performance. This is consistent with the general literature concerning self-reported and objective measures of cognitive functions in healthy participants (Martin \& Jones, 1983) and in schizophrenia (Stip et al. 2003; Chan et al. 2008). These findings suggest that PM is associated with the questionable segregation of cognitive functioning into two succinct domains (Chan et al. 2008).

By showing that it is a common impairment found in both schizophrenia and bipolar disorder, PM may represent a putative neuropsychological marker across the traditional diagnostic categorization. Along with the concept of continuous phenotype (Linscott \& van Os, 2010) and unitary psychosis (Crow, 1986), there have been several comparative studies recently conducted to examine certain neuropsychological functions other than PM in schizophrenia and bipolar disorder (for a review, see Glahn et al. 2007; Hill et al. 2008). These studies have demonstrated that both disorders shared similar neurocognitive impairments in phonological fluency, working memory, associative learning, verbal memory, attention and cognitive flexibility. Notably, our findings add to the existing literature by showing that such an overlap in neurocognitive deficits between schizophrenia and bipolar disorder could be extended to a new type of memory, namely, PM. It is therefore better to conceptualize the nosological boundary between schizophrenia and bipolar disorder as an ill-defined one, and to realize that the two clinical entities in fact share commonalities in a variety of areas, from phenomenology of symptoms to neuropsychological functions and perhaps even genetic architecture. Confining strictly to the Kraepelinian dichotomy instead might hamper a genuine understanding of the aetiology of psychosis, and unjustifiably limit the study designs, in terms of recruitment of suitable subjects and statistical power, of research for the susceptibility genes of psychosis.

\section{Limitations and implications}

Our study has several limitations. First, we did not ascertain whether the PM difference observed in this study is a primary deficit or confounded by group differences in other neurocognitive functions. In fact, prior literature has concluded that PM is correlated with cognitive flexibility, attention, working memory and response inhibition (Wang et al. 2009). Ideally, all such neurocognitive functions should be matched by careful recruitment of clinical and healthy samples. Alternatively, group differences in these neurocognitive functions should be controlled for using post-hoc covariate analysis. Second, it is important to note that bipolar disorder is a highly heterogeneous phenotype (Keshavan et al. 2011), which could be classified differently according to bipolarity, severity and lifetime history of psychosis. Glahn et al. (2006) used an interesting methodology by classifying their bipolar group into subgroups with and without lifetime history of psychosis, and found that the bipolar subgroup with psychosis shared a greater similarity in working memory impairment with schizophrenia than did the subgroup without psychosis. A similar method to refine the bipolar phenotype has been employed in other studies (Potash et al. 2001, 2003) showing overlaps of susceptibility genes across the Kraepelinian dichotomy. Our sample with bipolar disorder was heterogeneous and adopting such an analytical method might be suitable and useful. However, due to the small sample size $(n=9$ for bipolar disorder with history of psychotic symptoms; $n=31$ for bipolar disorder without history of psychotic symptoms), we could not employ the analysis used by Glahn et al. (2007).

In prior literature, time-based PM is usually thought to be more difficult than event-based PM because it requires a greater degree of self-initiation. However, our post-hoc results found the semantic event-based PM session the most difficult. It should be noted that task difficulty depends on many factors, such as the nature of event-based PM cues (focal or non-focal) and the regularity of the time-based PM task. Our laboratory PM assessment comprised different paradigms with variable task difficulty, and some of them, such as the activity-based PM, might be less cognitively demanding and might not be sensitive enough to identify potential impairment in our clinical samples.

Notwithstanding these limitations, the current study has several implications for future research in PM. We propose that future PM paradigms should be modified to include measurement of one's time monitoring, and evaluation of one's self-perception as to whether the PM task has been executed. A dual-task paradigm could provide a useful framework with which to examine possible defective internal sources monitoring (Elvevag et al. 2003; Smith, 2003) in individuals with schizophrenia and bipolar disorder. Elvevag et al. (2003) proposed that individuals with schizophrenia might fail to distinguish the intention to perform a task from the actual performance of the task. Furthermore, by varying the nature (time- or eventbased) of the PM tasks, it might be feasible to further distinguish defective temporal discrimination (i.e. monitoring of elapsing time in time-based PM) from defective source monitoring (i.e. distinguishing a PM cue on the screen from the stimuli in the ongoing task). Likewise, an improved research methodology to include larger samples with homogeneous psychosis 
phenotype and to measure and control for other neurocognitive functions is essential for us to re-examine these issues.

\section{Conclusions}

The current study has specifically examined the similarity and difference in PM between schizophrenia and bipolar disorder. It adds to the growing body of evidence for shared neurocognitive impairments between schizophrenia and bipolar disorder. Our findings challenge the tradition of Kraepelinian dichotomy and provide evidence to support the concept of continuum of psychosis. The findings of our study suggest that PM impairment might be a neuropsychological marker for psychosis, existing in both schizophrenia and bipolar disorder. The concept of continuous phenotype (Linscott \& van Os, 2010) and the endophenotype approach (Gottesman \& Gould, 2003) are viable strategies to elucidate phenotype heterogeneity. Neuropsychological markers, such as PM impairment, may provide a useful framework for us to understand the phenotype heterogeneity. From a clinical perspective, it is possible that certain common problems in patients with schizophrenia and bipolar disorder, such as forgetting to take medications or failing to attend an appointment, could be partly contributed by PM deficit and might be potentially modifiable in cognitive mediation therapy.

\section{Acknowledgements}

This study was supported partially by grants from the National Science Fund China (no. 81088001 and 91132701), a grant from the Knowledge Innovation Project of the Chinese Academy of Sciences (no. KSCX2-EW-J-8), the initiation fund of the China Academy of Sciences and State Administration of Foreign Experts Affairs of the People's Republic of China (CAS/SAFEA, Y2CX131003) International Partnership Program for Creative Research Teams, and the Smart Futures Fund (QLD), National and International Research Alliances Programme.

\section{Declaration of Interest}

None.

\section{References}

Altgassen M, Kliegel M, Rendell P, Henry JD, Zollig J (2008). Prospective memory in schizophrenia: the impact of varying retrospective-memory load. Journal of Clinical and Experimental Neuropsychology 30, 777-788.
Arts B, Jabben N, Krabbendam L, van Os J (2008). Metaanalyses of cognitive functioning in euthymic bipolar patients and their first-degree relatives. Psychological Medicine 38, 771-785.

Braff DL (1993). Information processing and attention dysfunctions in schizophrenia. Schizophrenia Bulletin 19, 233-259.

British Medical Association and Royal Pharmaceutical Society of Great Britain (2009). British National Formulary: 58: September 2009. British Medical Association and Royal Pharmaceutical Society of Great Britain: London.

Burgess PW, Shallice T (1996a). Response suppression, initiation and strategy use following frontal lobe lesions. Neuropsychologia 34, 263-273.

Burgess PW, Shallice T (1996b). Bizarre responses, rule detection, and frontal lobe lesions. Cortex 32, 241-259.

Chan RCK, Chen EYH, Cheung EFC, Chen RYL, Cheung HK (2006a). The components of executive functioning in a cohort of patients with chronic schizophrenia: a multiple single-case study design. Schizophrenia Research 81, 173-189.

Chan RCK, Chen EYH, Cheung EFC, Cheung HK (2004). Executive dysfunctions in schizophrenia: relationships to clinical manifestations. European Archives of Psychiatry and Clinical Neuroscience 254, 256-262.

Chan RCK, Chen EYH, Law CW (2006b). Specific executive dysfunction in patients with first-episode medication-naïve schizophrenia. Schizophrenia Research 82, 51-64.

Chan RCK, Wang Y, Ma Z, Hong X, Yuan Y, Yu X, Li Z, Shum D, Qong QY (2008). Objective measures of prospective memory do not correlate with subjective complaints in schizophrenia. Schizophrenia Research 103, 229-239.

Crow T (1986). The continuum of psychosis and its implication for the structure of the gene. British Journal of Psychiatry 149, 419-429.

Einstein GO, McDaniel MA (1990). Normal aging and prospective memory. Journal of Experimental Psychology Learning Memory and Cognition 16, 717-726.

Einstein GO, McDaniel MA (1996). Retrieval processes in prospective memory: theoretical approaches and some new empirical findings. In Prospective Memory: Theory and Applications (ed. M. A. Brandimonte, G. O. Einstein and M. A. McDaniel), pp. 115-142. Lawrence Erlbaum Associates: Mahwah, NJ.

Ellis JA (1996). Prospective memory or the realization of delayed intentions: a conceptual framework for research. In Prospective Memory: Theory and Applications (ed. M. A. Brandimonte, G. O. Einstein and M. A. McDaniel), pp. 1-22. Lawrence Erlbaum Associates: Mahwah, NJ.

Ellison-Wright I, Bullmore E (2010). Anatomy of bipolar disorder and schizophrenia: a meta-analysis. Schizophrenia Research 117, 1-12.

Elvevag B, Maylor EA, Gilbert AL (2003). Habitual prospective memory in schizophrenia. BMC Psychiatry 3, 9.

Glahn DC, Bearden CE, Barguil M, Barrett J, Reichenberg A, Bowden CL, Soares JC, Velligan DI (2007). The 
neurocognitive signature of psychotic bipolar disorder. Biological Psychiatry 62, 910-916.

Glahn DC, Bearden CE, Cakir S, Barrett JA, Najt P, Monkul ES, Maples N, Velligan DI, Soares JC (2006). Differential working memory impairment in bipolar disorder and schizophrenia: effects of lifetime history of psychosis. Bipolar Disorder 8, 117-123.

Gold JM, Carpenter C, Randolph C, Goldberg TE, Weinberger DR (1997). Auditory working memory and Wisconsin Card Sorting Test performance in schizophrenia. Archives of General Psychiatry 54, 159-165.

Gong YX (1992). Manual of Wechsler Adult Intelligence ScaleChinese Version. Chinese Map Press: Changsha.

Gottesman II, Gould TD (2003). The endophenotype concept in psychiatry: etymology and strategic intentions. American Journal of Psychiatry 160, 636-645.

Hamilton M (1960). A rating scale for depression. Journal of Neurology, Neurosurgery, and Psychiatry 23, 56-62.

Heaton RK, Gladsjo JA, Palmer BW, Kuck J, Marcotte TD, Jeste DV (2001). Stability and course of neuropsychological deficits in schizophrenia. Archives of General Psychiatry 58, 24-32.

Heinrichs RW, Zakzanis KK (1998). Neurocognitive deficit in schizophrenia: a quantitative review of the evidence. Neuropsychology 12, 426-445.

Hill SK, Harris MS, Herbener ES, Pavuluri M, Sweeney JA (2008). Neurocognitive allied phenotypes for schizophrenia and bipolar disorder. Schizophrenia Bulletin 34, 743-759.

Hung GBK (2007). A comparison of two methods in calculating total antipsychotics dosage. Hong Kong Journal of Psychiatry 17, 87-90.

Ivleva EI, Morris DW, Moates AF, Suppes T, Thaker GK, Tamminga CA (2010). Genetics and intermediate phenotypes of the schizophrenia-bipolar disorder boundary. Neuroscience, Biobehavioral Reviews 34, 897-921.

Kay SR, Fiszbein A, Opler LA (1987). The Positive and Negative Syndrome Scale (PANSS) for schizophrenia. Schizophrenia Bulletin 13, 261-276.

Keshavan MS, Morris DW, Sweeney JA, Pearlson G, Thaker G, Seidman LJ, Eack SM, Tamminga C (2011). A dimensional approach to the psychosis spectrum between bipolar disorder and schizophrenia: the Schizo-Bipolar Scale. Schizophrenia Research 12, 250-254.

Kliegel M, Altgassen M, Hering A, Rose NS (2011). A process-model based approach to prospective memory impairment in Parkinson's disease. Neuropsychologia 49, 2166-2177.

Kliegel M, Jager T, Altgassen M, Shum D (2008a). Clinical neuropsychology of prospective memory. In Prospective Memory: Cognitive, Neuroscience, Developmental, and Applied Perspectives (ed. M. Kliegel, M. A. McDaniel and G. O. Einstein), pp. 283-308. Lawrence Erlbaum Associates: New York.

Kliegel M, Jager T, Phillips LH (2008b). Adult age differences in event-based prospective memory: a meta-analysis on the role of focal versus nonfocal cues. Psychology and Aging 23, 203-208.
Kvavilashvili L (1992). Remembering intentions: a critical review of existing experimental paradigms. Applied Cognitive Psychology 6, 507-524.

Kvavilashvili L, Ellis JA (1996). Variety of intentions: some distinctions and classifications. In Prospective Memory: Theory and Applications (ed. M. A. Brandimonte, G. O. Einstein and M. A. McDaniel), pp. 23-52. Lawrence Erlbaum: Mahwah, NJ.

Lee E, Xiang YT, Man D, Au RWC, Shum D, Tang WK, Chiu HFK, Wong P, Ungvari GS (2010). Prospective memory deficits in patients with bipolar disorder: a preliminary study. Archives of Clinical Neuropsychology 25, 640-647.

Linscott RJ, van Os J (2010). Systematic review of categorical versus continuum model in psychosis: evidence for discontinuous subpopulations underlying a psychometric continuum. Implications for DSM-V, DSM-VI and DSM-VII. Annual Review of Clinical Psychology 6, 391-419.

Lui SSY, Wang Y, Liu ACY, Chui WWH, Gong Q-Y, Shum D, Cheung EFC, Chan RCK (2011). Prospective memory in patients with first-onset schizophrenia and their non-psychotic siblings. Neuropsychologia 49, 2217-2224.

Martin M, Jones GV (1983). Cognitive failures in everyday life. In Everyday Memory, Actions and Absent-mindedness (ed. J. E. Harris and P. E. Morris), pp. 173-190. Academic Press: London.

Norman DA, Shallice T (1986). Attention to action: willed and automatic control of behaviour. In Consciousness and Self-Regulation: Advances in Research and Theory, vol. 4 (ed. R. J. Davidson, G. E. Schwartz and D. Shapiro), pp. 1-18. Plenum: New York.

Potash JB, Bienvenu OJ (2009). Neuropsychiatric disorders: shared genetics of bipolar disorder and schizophrenia. Nature Reviews Neurology 5, 299-300.

Potash JB, Willour VL, Chiu YF, Simpson SG, MacKinnon DF, Pearlson GD, Depaulo JR, Mcinnis MG (2001). The familial aggregation of psychotic symptoms in bipolar disorder pedigrees. American Journal of Psychiatry 158, 1258-1264.

Potash JB, Zandi PP, Willour VL, Lan T-H, Huo Y, Avramopoulos D, Shugart YY, MacKinnon DF, Dean F, Simpson SG, McMahon FJ, Depaulo JR, McInnis MG (2003). Suggestive linkage to chromosomal regions 13q31 and 22q12 in families with psychotic bipolar disorder. American Journal of Psychiatry 160, 680-686.

Shifman S, Bronstein M, Sternfeld M, Pisante A, Weizman A, Reznik I, Spivak B, Grisaru N, Karp L, Schiffer R, Kotler M, Strous RD, Swartz-Vanetik M, Knobler HY, Shinar E, Yakir B, Zak NB, Darvasi A (2004). COMT: a common susceptibility gene in bipolar disorder and schizophrenia. American Journal of Medical Genetics Part B 128B, 61-64.

Shum D, Ungvari GS, Tang WK, Leung JP (2004). Performance of schizophrenia patients on time-, event-, and activity-based prospective memory tasks. Schizophrenia Bulletin 30, 693-701.

Smith G, della Sala S, Logie RH, Maylor EA (2000). Prospective memory and retrospective memory in normal 
ageing and dementia: a questionnaire study. Memory 8 , 311-321.

Smith RE (2003). The cost of remembering to remember in event-based prospective memory: investigating the capacity demands of delayed intention performance. Journal of Experimental Psychology: Learning, Memory, and Cognition 29, 347-361.

Spreen O, Strauss E (1991). A Compendium of Neuropsychological Tests: Administration, Norms, and Commentary. Oxford University Press: New York.

Stip E, Caron J, Renaud S, Pampoulova T, Lecomte Y (2003). Exploring cognitive complaints in schizophrenia: the subjective scale to investigate cognition in schizophrenia. Comprehensive Psychiatry 44, 331-340.

Tamlyn D, McKenna PJ, Mortimer AM, Lund CE, Hammond S, Baddeley AD (1992). Memory impairment in schizophrenia: its extent, affiliations and neuropsychological character. Psychological Medicine 22, 101-115.

Wang Y, Chan RCK, Cui J, Deng Y, Huang J, Li H, Yan C, Xu T, Ma Z, Hong X, Li Z, Shi H, Shum D (2010).

Prospective memory in non-psychotic first-degree relatives of patients with schizophrenia. Psychiatry Research 179, 285-290.

Wang Y, Chan RCK, Hong X, Ma Z, Yang T, Guo L, Yu X, Li Z, Yuan Y, Qong GY, Shum D (2008a). Prospective memory in schizophrenia : further clarification of nature of impairment. Schizophrenia Research 105, 114-124.
Wang Y, Chan RCK, Yu X, Shi C, Cui J, Deng Y (2008b). Prospective memory deficits in subjects with schizophrenia spectrum disorders: a comparison study with schizophrenic subjects, psychometrically defined schizotypal subjects, and healthy controls. Schizophrenia Research 106, 70-80.

Wang Y, Cui J, Chan RCK, Deng Y, Shi H, Hong X, Li Z, Yu X, Qong GY, Shum D (2009). Meta-analysis of prospective memory in schizophrenia : nature, extent, and correlates. Schizophrenia Research 114, 64-70.

WHO (1992). The ICD-10 Classification of Mental and Behavioural Disorders: Clinical Descriptions and Diagnostic Guidelines. World Health Organization: Geneva.

Young RC, Biggs JT, Ziegler VE, Meyer DA (1978). A rating scale for mania: reliability, validity and sensitivity. British Journal of Psychiatry 133, 429-435.

Yu K, Cheung C, Leung M, Li Q, Chua S, McAlonan G (2010). Are bipolar disorder and schizophrenia neuroanatomically distinct? An anatomical likelihood meta-analysis. Frontiers in Human Neuroscience 4, 189.

Zeintl M, Kliegel M, Hofer SM (2007). The role of processing resources in age-related prospective and retrospective memory within old age. Psychology and Aging 22, 826-834.

Zhou F, Xiang Y, Wang C, Dickerson F, Au RCK, Zhou J, Shum DHK, Chiu HFK, Man D, Lee EHM, Yu X, Chan RCK, Ungvari GS (2012). Characteristics and clinical correlates of prospective memory performance in firstepisode schizophrenia. Schizophrenia Research 135, 34-39. 